INPLASY

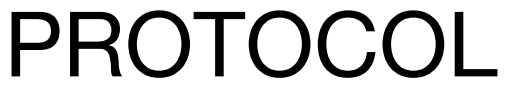

To cite: Zheng et al.

Acupuncture for knee osteoarthritis with depression:

A protocol for systematic review and meta analysis. Inplasy protocol 2021120132. doi:

10.37766/inplasy2021.12.0132

Received: 29 December 2021

Published: 29 December 2021

Corresponding author:

Xu Zheng

yiyuran88@163.com

Author Affiliation:

None

Support: 20200403123SF.

Review Stage at time of this submission: The review has not yet started.

Conflicts of interest:

None declared.

\section{Acupuncture for knee osteoarthritis with depression: A protocol for systematic review and meta analysis}

\author{
Zheng, X1; Liu, Y2; Li, $\mathrm{H}^{3}$.
}

Review question / Objective: Depression and obesity are highly comorbid among knee $O A$ patients, and the combination of obesity and depression is associated with decreased physical activity, higher pain and disability, and more rapid cartilage degradation.This research aims to systematically and comprehensively evaluate the safety and efficacy of acupuncture in the treatment of KOA with depression.

Condition being studied: Knee osteoarthritis (OA) is the most common joint disorder and a leading cause of disability. Depression is highly comorbid among knee OA patients, and depression is associated with decreased physical activity, higher pain and disability, and more rapid cartilage degradation. Depression, $O A$ exacerbate one another and share a common pathophysiology involving systemic inflammation and pro-inflammatory cytokines, reflecting a complex mind-body interaction. Current treatments for knee OA offer little to no benefit over placebo.

INPLASY registration number: This protocol was registered with the International Platform of Registered Systematic Review and Meta-Analysis Protocols (INPLASY) on 29 December 2021 and was last updated on 29 December 2021 (registration number INPLASY2021120132).

\section{INTRODUCTION}

Review question / Objective: Depression and obesity are highly comorbid among knee OA patients, and the combination of obesity and depression is associated with decreased physical activity, higher pain and disability, and more rapid cartilage
degradation.This research aims to systematically and comprehensively evaluate the safety and efficacy of acupuncture in the treatment of KOA with depression.

Condition being studied: Knee osteoarthritis (OA) is the most common 
joint disorder and a leading cause of disability. Depression is highly comorbid among knee OA patients, and depression is associated with decreased physical activity, higher pain and disability, and more rapid cartilage degradation. Depression, $O A$ exacerbate one another and share a common pathophysiology involving systemic inflammation and proinflammatory cytokines, reflecting a complex mind-body interaction. Current treatments for knee OA offer little to no benefit over placebo.

\section{METHODS}

Participant or population: The patients of knee OA with depression.

Intervention: Acupuncture.

Comparator: Therapies other than acupuncture (medicine, tuina, external therapy).

Study designs to be included: All randomized controlled trials (RCT) and quasi-RCTs study on acupuncture in the treatment of KOA with depression will be included.

Eligibility criteria: 1.All patients included in the study were diagnosed in KOA with depression, regardless of age, gender, race. 2.The trail group uses acupuncture or combination therapy with other treatments. The acupuncture method, acupoint selection, and needles are not limited; the control group uses the intra-articular glucocorticoid injections, and other adjuvant treatments can be appropriately added. The trail group and the control group are not limited in terms of medication, dosage, and treatment course.

Information sources: The following 8 electronic databases will be searched, including PubMed, Embase, the Cochrane Library, China National Knowledge Infrastructure (CNKI), Web of Science, Chinese Scientific Journal Database (VIP), Wanfang Database, and Chinese Biomedical Literatures Database (CBM) without any restrictions.
Main outcome(s): The primary outcome measure is Western Ontario and McMaster Universities Arthritis Index (WOMAC), which was divided into pain score, stiffness score, and daily activity score.

Quality assessment / Risk of bias analysis: Two researchers will conduct the assessment independently via using Cochrane Collaboration's risk of bias tool, which mainly involved sequence generation, allocation concealment, blinding, incomplete outcome data, selective outcome reporting, and other sources of bias. Similarly, we will resolve the disagreements arising from the evaluation process via discussion, and a third assessor will intervene and arbitrate. Generally, the RoB of high-quality RCTs has little impact on the results of the study. However, if the quality of included RCTs is uneven, the possible impact of RoB on the total effect should be analyzed, or only the results with low RoB will be combined.

Strategy of data synthesis: We will use Review Manger V.5.3 software (Cochrane, London, UK) to conduct meta-analysis, and the results will be presented in the form of forest maps. If the result of overall effect test is $\mathrm{P}<.05$, it means that difference between the experimental group and control group is statistically significant. On the contrary, the difference is not statistically significant. As for the index of effect size, we will use the standardized mean difference to evaluate the continuous variables, while using rate ratio for dichotomous variables. The confidence intervals for both continuous and dichotomous variables will be set to $95 \%$.

Subgroup analysis: In the condition of obvious heterogeneity and adequate trials, we will conduct the subgroup analysis to analyze the source of heterogeneity, on the basis of difference in interventions, controls, participant characteristics, course of treatment, dose gradients, and outcome measures. For instance, included trials were divided into 2 subgroups basis of different dose gradients, and the 2 subgroups were proved to be homogeneous, respectively, via 
heterogeneity test. It indicated that dose factor was the source of heterogeneity if the data of the 2 subgroups were combined and detected significant heterogeneity. On the contrary, it showed that the dose has little influence on the research results.

Sensitivity analysis: The combination of different research data is bound to produce heterogeneity. By excluding the included literature, respectively, we will analyze the changes of heterogeneity and other merger effects after excluding the corresponding literature, and infer the literature that causes heterogeneity. Then, from the design of the experiment, sample size, outcome indicators, and other aspects, we will analyze why the RCT is the source of heterogeneity. However, if the heterogeneity results are stable after excluding the literature, respectively, our research results can be considered as reliable.

Country(ies) involved: China.

Keywords: knee osteoarthritis with depression, protocol, systematic review and meta-analysis, acupuncture.

Contributions of each author:

Author 1 - Xu Zheng.

Author 2 - Yang Liu.

Author 3 - Huijing Li. 